\title{
Removal of misfit dislocations from mismatched layers in heterostructures
}

\author{
Mileta M Arakelyan* \\ Chair of Solid-state physics, Department of Physics of the Yerevan State University, \\ 1, Alex Manoogian St., Yerevan, 0025, Armenia
}

\begin{abstract}
The method of the misfit dislocation removal from working area of the devices on heterostructures is theoretically offered. The chainlet of the edge dislocation (one-dimensional soliton-soliton bound state) is formed in substrate and film border by special preliminary processing. The stress field of such chainlet pushes away a misfit dislocation incipient on the borders of the heterostructures. By numerical experiment it is shown that the stress field of a chainlet is compressed in a sliding direction and it increases in a perpendicular direction, when the velocity is increased. It is possible to influence locally on the given dislocation varying the parameters of the system.
\end{abstract}

Keywords: misfit dislocation, topological soliton, two-soliton chain, heterostructure.

\section{INTRODUCTION}

Thin-film structures are widely used in nanoelectronics. The difference between the lattice parameters in these structures causes the appearance of the internal stresses and misfit dislocations in the films, which worsen the functional properties of the heterostructures ${ }^{1}$. For the prevention of premature failure of devices on heterostructures it is necessary to remove the dislocations from the active area of devices.

One of the methods of solving this problem is a plastic deformation of the substrate leading to the formation of the walls of the edge dislocation, which block the initiation of misfit dislocations in the heterostructures ${ }^{2}$. Y. Obayashi and Shintani K offered to form thin buffer layers with the set structure on the border between films and substrates to increase the critical thickness of films on substrates ${ }^{3}$. I.W. Matthews and A.E. Blakeslee offered to remove the dislocations from epitaxial films through lateral sides under the action of the misfit stress ${ }^{4}$. M. Gutkin and I.A Ju. Obid'ko studied the theoretical models of interfaces and misfit defects in polycrystalline and nanocrystalline films and the questions of the faceting of grain boundary in thin films under the action of the misfit stress ${ }^{5}$. According to Zhang X.G., Rodriguez A. Wang X., Li P, Jain F.C., Ayers J.E the threading dislocations can be removed in patterned heteroepitaxial processing by glide to the sidewalls, as promoted by the presence of image forces ${ }^{6}$. I.A. Ovid'ko proposed a theoretical model which describes a new physical micromechanizm for relaxation of misfit stresses in crystalline films, namely the formation of misfit dislocation walls ${ }^{7}$.

This work offers the method of the cleaning of the device working area from the misfit dislocations: the chainlets of the edge dislocations are formed in the substrate, which by their stress field block or push away the misfit dislocations. All the results were obtained by a numerical experiment.

Solitons are nonlinear structures which carry the important information about properties of the medium. The model of the edge dislocation, proposed by Frenkel and Kontorova, works well at the investigation of the epitaxial heterostructures and makes possible it for a quantitative analys.

Frenkel-Kontorowa dislocations are topological solitons or structural defects of order parameter; their movement can be described by one dimensional sine - Gordon equation ${ }^{8}$ :

$$
\varphi_{t t}-c_{0}^{2} \varphi_{x x}+\omega_{0}^{2} \sin \varphi=0,
$$

\footnotetext{
*-mail: marakelyan@ysu.am
}

Photonics and Micro- and Nano-structured Materials 2011, edited by Rafael Kh. Drampyan,

Proc. of SPIE Vol. 8414, 84140K · C 2012 SPIE · CCC code: 0277-786X/12/\$18 - doi: 10.1117/12.923228 
where $c_{0}$ is the speed of sound, $\omega_{0}^{2} \equiv \frac{2 \pi f_{0}}{m a}$ is characteristic frequency, $f=f_{0} \sin \left(\frac{2 \pi x}{a}\right)$ is a periodic force acting from the side of the wafer in the models of Frenkel - Kontorova, $a$ is a lattice constant. The solution of the unperturbed sine-Gordon equation has a form ${ }^{8}$ :

$$
\varphi(x, t)=\pi-4 \operatorname{arctg}\left[\exp \left(\frac{x-v t}{l}\right)\right],
$$

where $l=\frac{v}{\omega_{0}}$ is the half-width of the dislocation, $v$ is the velocity of the dislocation.

The bound states soliton-soliton, soliton-antisoliton are the fundamental solutions of equation (1), in addition to solution (2).

As it is generally known ${ }^{9}$, solitons interacting with periodic potential of the lattice or soliton-soliton bound states are adequate to the kink crossing potential barriers. At $\mathrm{T} \leq 50 \mathrm{~K}$ the applied mechanical stress causes motion of single kinks, and the thermally activated formation of soliton-antisoliton couples takes place at more high temperatures. In crystals the chainlets of solitons of one sign can be obtained by means of the known technologies 9. (A sample is subject to the large plastic deformation and then is maintained during a few twenty-four hours at a room temperature. As a result the disorderly interlace dislocation accumulations disintegrate and a certain part of dislocation is oriented under small corners to crystallography directions).

We have simulated the receipt of two-soliton chainlet and investigated its influence on the motion of the single dislocation.

\section{INTERACTION OF A SINGLE DISLOCATION WITH THE DISLOCATION CHAINLET}

The bound state of two solitons is possible to be considered as dislocation chainlet. We investigated the interaction of a moving single Frenkel-Kontorowa dislocation with an elastic field of the dislocation chainlet at their combined motion at the same speed in one gliding plane. A member describing such field is added to the sine - Gordon equation, and by numerical experiment the influence of this field on a single dislocation is investigated at different velocities of the cooperative motion.

Two-soliton solution of unperturbed equation of a sine- Gordon in dimensionless units is ${ }^{8}$ :

$$
\begin{aligned}
& \varphi(x, t)=4 \operatorname{arctg}\left[\left\{1-\frac{1-u_{1} u_{2}-\sqrt{\left(1-\left(u_{1}\right)^{2}\right.} \sqrt{\left(1-\left(u_{2}\right)^{2}\right.}}{1-u_{1} u_{2}+\sqrt{\left(1-\left(u_{1}\right)^{2}\right.} \sqrt{\left(1-\left(u_{2}\right)^{2}\right.}} \operatorname{Exp}\left[-\frac{x-x_{1}-u_{1} t}{\sqrt{\left(1-\left(u_{1}\right)^{2}\right)}}-\frac{x-x_{2}-u_{2} t}{\sqrt{\left(1-\left(u_{2}\right)^{2}\right)}}\right]\right\} /\right. \\
& \left.\left(\operatorname{Exp}\left[-\frac{x-x_{1}-u_{1} t}{\sqrt{\left(1-\left(u_{1}\right)^{2}\right)}}\right]+\operatorname{Exp}\left[-\frac{x-x_{2}-u_{2} t}{\sqrt{\left(1-\left(u_{2}\right)^{2}\right.}}\right]\right)\right],
\end{aligned}
$$

where $u_{1}, u_{2}$ are velocities, $x_{1}, x_{2}$ are initial coordinates, $\varphi$ is deviation of atom from position of equilibrium, measured in the angular units. At a certain moment of time the respective plot can be presented as:

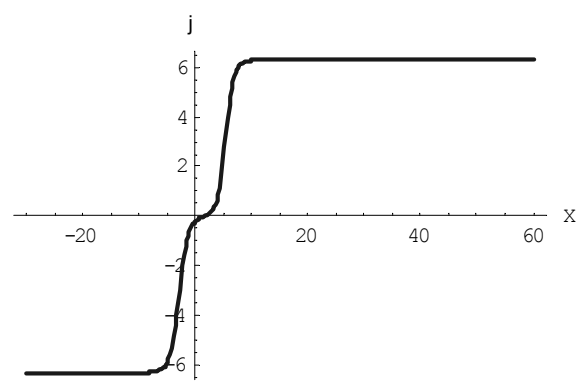

Figure 1. The displacement field of the two-soliton chainlet. 
The equation of a sine- Gordon changes to the equation given below if there is perturbation from the side of dislocation chainlet:

$$
\varphi_{t t}-c_{0}^{2} \varphi_{x x}+\omega_{0}^{2} \sin \varphi-\frac{2 \pi F_{x}}{m a}=0
$$

$F_{x}$ is a projection of force acting on the length unit of dislocation from the side of the dislocation chain. Only the shear stresses $\sigma_{x y}$ act in the gliding plane (normal stresses equal zero: $\sigma_{x x}=0, \sigma_{y y}=0, \sigma_{z z}=0$ ). The strain is a derivative of displacement and it determines the components of stress and force:

$$
\varepsilon_{x}=\frac{\partial \varphi}{\partial x}, \sigma_{x}=E \varepsilon_{x}, F_{x}=b \sigma_{x},
$$

where $\varepsilon_{x}$ is a component of strain, $\sigma_{x}$ is a component of the shear stress, $b$ is the Burgers vector, $E$ is the Youngs modulus.

The act of the field of two-soliton chainlet on single dislocation depending on the velocity of their motion was investigated by a numerical experiment. At the absence of the interaction cooperative motion of such structure for the different moments of time is presented on Fig.1.

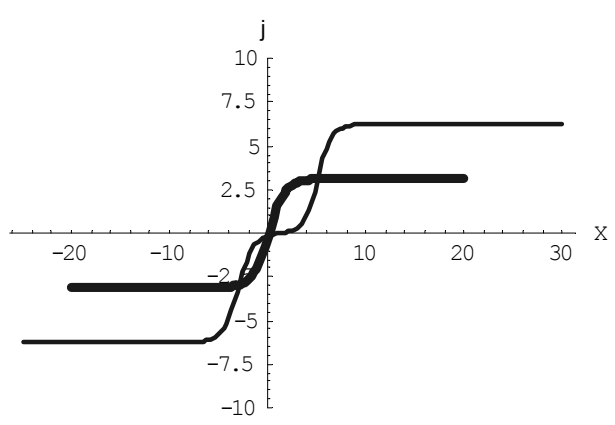

(a)

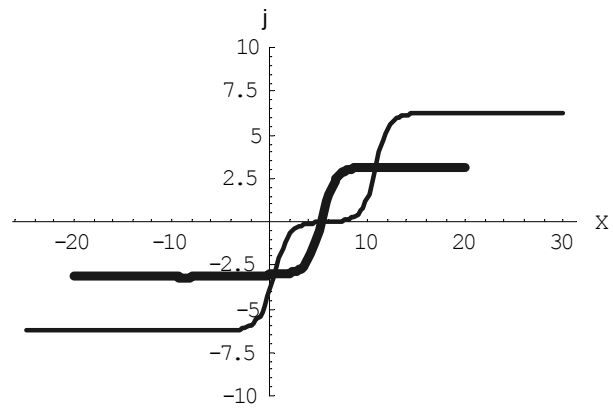

(b)

Figure.2. The fields of displacements of dislocation chainlet (thin line) and single dislocation (bold line) at the absence of the interaction between them at different consequent moments of time: (a) $t=t_{1}$; (b) $t=t_{2}>t_{1}$.

As Fig. 2 shows, single dislocation and twosolitonic chainlet move at the same speed, not interacting with each other. A numeral experiment suggests the following result at the elastic interaction of dislocation with dislocation chainlet. Fig.3.,Fig.4

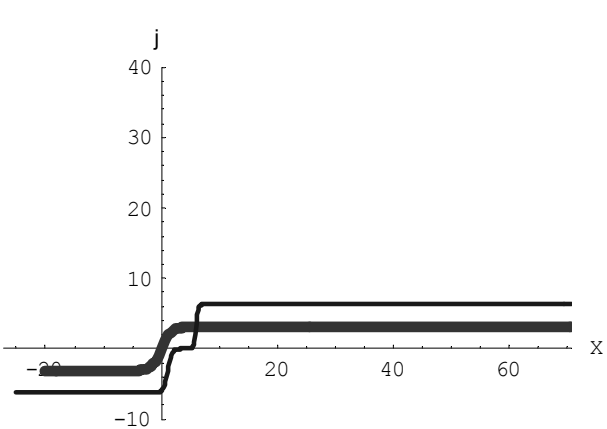

(a)

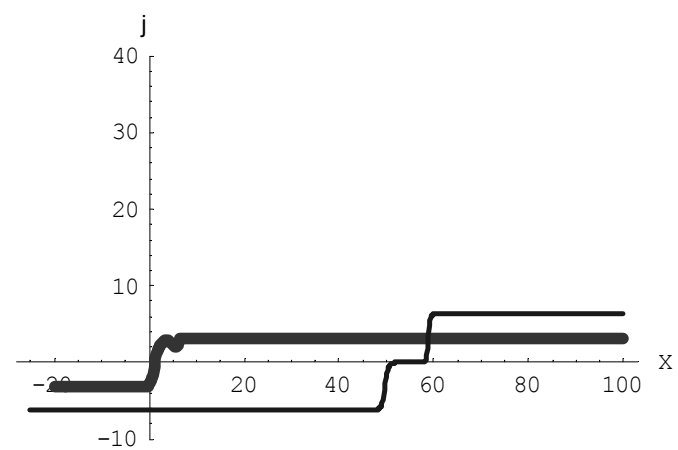

(b)

Figure 3. The displacements fields of interactive two-soliton chainlet (thin line) and single dislocation (bold line) at a high velocity of sliding at the same moments of time: (a) $t=t_{1}$; (b) $t=t_{2}>t_{1}$. 


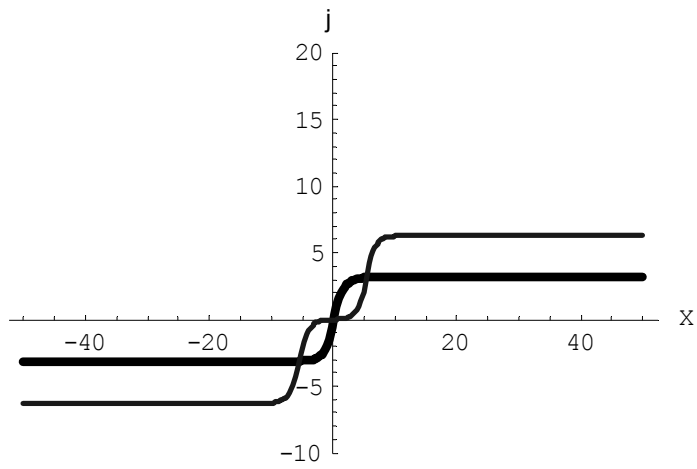

(a)

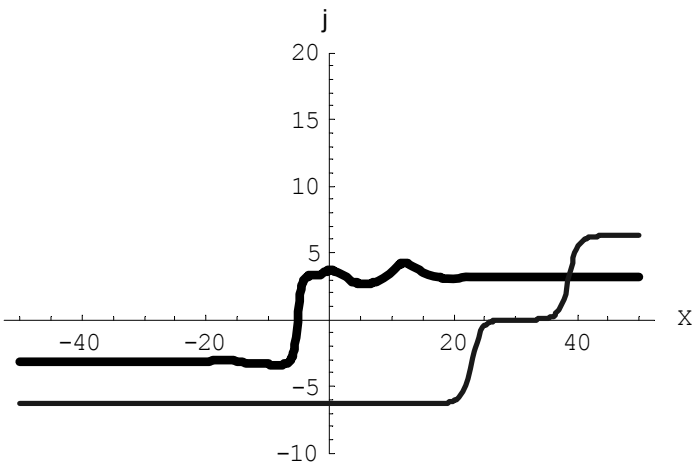

(b)

Figure 4. The fields of displacements of interactive two-soliton chainlet (thin line) and single dislocation (bold line) at a low velocity of sliding at the same moments of time: (a) $t=t_{1}$; (b) $t=t_{2}>t_{1}$.

As it is seen from comparison of Fig. 3 and Fig. 4 the repulsive force increases with the decrease of speed: two-soliton chainlet blocks the motion of the single dislocation at a high velocity of sliding (Fig.3) and it pushes away the single dislocation at a low velocity of sliding (Fig.4).

To explain the received effect the stress fields of the dislocation chainlets were simulated at considered different velocities of cooperative motion $\left(\sim 10^{-2} \frac{\mathrm{cm}}{\mathrm{sec}} \div 10^{3} \frac{\mathrm{cm}}{\mathrm{sec}}\right)$. Fig. 5 .

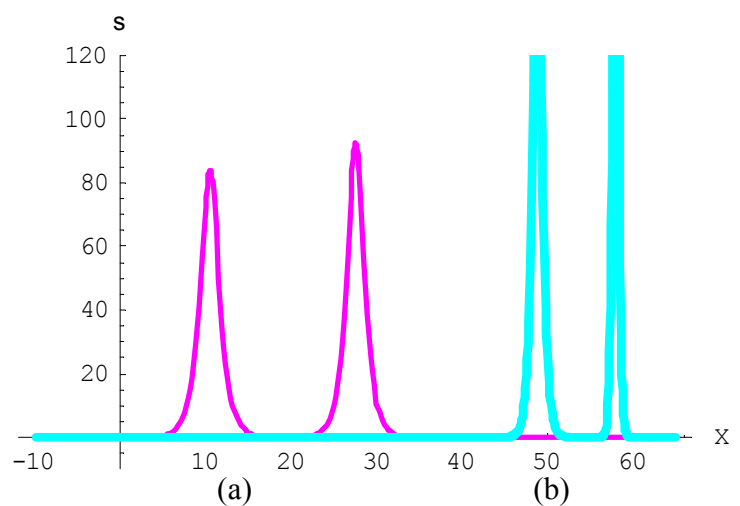

Figure 5. Fields of stresses of dislocation chainlet at the investigated speeds of sliding in the same moment of time.

(a) low velocity of sliding; (b) high velocity of sliding.

A numerical experiment suggests (Fig.5), that a dislocation chainlet stress field is compressed in the glide direction when the velocity is increased. The leading edge of the stress field of the dislocation chain is increased in amplitude. And, hence, in the slip plane the interaction between the dislocation and dislocation chain decreases with the increase of slip velocity. A numerical experiment suggests that in the limit, when the velocity tends to the sound speed, the interaction between them tends to zero.

Half-infinite wafer with the chainlet of solitons and film are practically used. The film and the wafer are elasticisotropic solids with the identical shear modulus and Poisson ratio. The wafer and the film have identical types of crystal lattice, but different lattice constant.

We recommend to use heterostructures $\mathrm{Co} / \mathrm{Cu}$ in which the critical thickness is sufficiently small, and linear density of misfit dislocations is great (reaches $1,3 * 10^{6} \mathrm{~cm}^{-1}$ ) ${ }^{10}$. The misfit dislocations in $\mathrm{Fe} / \mathrm{Cu}$ and other heterostructures with substrates from face-centered cubic metals for which the big density of dislocations is characteristic, can also be used ${ }^{11}$.

All constants are taken for a copper ${ }^{12}$. The copper was preferred because the motion of dislocations in copper (face-centered cubic structure) begins at very low values of stresses (it is $8 * 10^{2} \mathrm{~cm} / \mathrm{sec}$ at $298 \mathrm{~K}$ for $\sigma \approx 10^{6} \mathrm{dyn} / \mathrm{cm}^{2}$ ) and may vary as a function of the stress in a wide range of velocities. 


\section{CONCLUSION}

The action of elastic field of two-soliton chainlet on the single misfit dislocation is investigated. It is shown, that if these structures are in the same glide planes then at a low velocity of sliding their interaction is stronger than that at a high velocities. The numerical experiment showed that the stress fields of both two-soliton chainlet and single dislocation are compressed in the glide direction at the increase of the velocity.

The presence of dislocation chainlet blocks dislocation motion and it is a stopper repulsing the dislocation. The dislocation is pushed by the field of the dislocation chainlet, and the repulsive force in an investigated velocity range decreases with the velocity increase. It is possible to regulate this action varying the parameters of the system. The presence of the multisolitonic chainlet results in the following: on a border film-wafer, the multisolitonic chainlet pushes off the misfit dislocations from the working area of heterostructures, which secures the stability of properties of such structures.

\section{REFERENCES}

[1] Tkhorik, Ju. A. Hazan, L.S. [Plastic deformation and misfit dislocations in the heteroepitaxial systems], Naukowa dumka, Kiev, (1983).

[2] Ovid'ko, I.,A., Shejnerman, A.,G.,"Misfit dislocation in thin films on plastically deformed substrates". Fizika twerdogo tela, 44(7), 1243-1248 (2002).

[3] Obayashi Y., Shintani K., J.Appl.Phys., 88 (10), 105 (2000).

[4] Matthews I.W., Blakeslee A.E. "Defects in epitaxial multiplayers". J.Crysy.Growth, 32(2), 265-273 (1976).

[5] Gutkin M.Ju. Obid'ko I.A. "Defect structures on internal interfaces in nanocrystalline and polycrystalline films", Materials Physics and Mechanics, 8, 108-148, (2009).

[6] Zhang X.G., Rodriguez A. Wang X., Li P, Jain F.C., Ayers J.E."Complete removal of threading dislocations from mismatched layers by patterned heteroepitaxial processing". Applied Physics Letters, 77(16), 2524-2526 (2000).

[7] Ovid'ko I.A. "Misfit dislocation walls in solid films". J.Phys.:Condens. Matter, 11, 6521-6527 (1999).

[8] Edited by Lonngren,K., Scott, A. Academic Press. [Soliton in Action], Proceedings of a Workshop, Academic Press, New York, San Francisko, London, 211-213 (1978).

[9] Melik-Shahnazarow, W., A., Mirzoewa, I.,I., Naskidashwili, I.,A.,’Tunneling of the dislocation kink in aluminium". Pis'ma v JETF, 43(5), 247-249 (1986).

[10] Fedorenko A.I., Vincent R.”The epitaxial growth of cobalt on copper”. Phil. Mag.,24(187), 55-62 (1971).

[11] Yagi k., Takaynagi K., Kobayashi K., Nonio G.'In situ observation of formation of misfit dislocations in pseudomorphic monolayer overgrowth of metals and non-metals". J.Cryst.Growth,9(1), 84-97 (1971).

[12] Hirt, J., Lote, I., [Theory of Dislocations], McGraw-Hill Book Company, New York, St.Louis, 168 (1972). 